\title{
Beam Cutting Effect on Jamming Efficiency of Chaff Corridor
}

\author{
Wang Hong-wei ${ }^{1, a}$, Wang Guo-wei ${ }^{2, b}$, Wang Hong-xun ${ }^{2, c}$, Chen You ${ }^{2, d}$, \\ Zhang $\mathrm{Xi}^{2, \mathrm{e}}$ \\ ${ }^{1}$ Electronic \& Information School, Northwestern Polytechnical University, Xi'an, Shaanxi, China \\ ${ }^{2}$ Aeronautics \& Astronautics Engineering School, AFEU, Xi'an, Shaanxi, China \\ ahww0818@163.com, 모73480178@qq.com, ${ }^{\mathrm{c} w h x w h x w h x @ 126 . c o m, ~}{ }^{\mathrm{d}}$ skyfishuu@163.com, ${ }^{\mathrm{e} z h a n}$ \\ gxiafeu@163.com
}

Keywords: Chaff Corridor, Blanket Efficiency, Jamming, Cutting effect.

\begin{abstract}
Cutting effect was unconsidered so far for Chaff Corridor (CC) both in theoretic analysis and operational engineering; different position's Blanket Efficiency (BE) of constant chaff-density CC is different as results of radar-lobes cut a chaff volume, which means there are limited chaffs which have jamming effect to radar. So CC's Jamming BE was researched and modified, even and un-even CC's chaff density of jamming efficiency were turned out by simulation, the results showed that if CC's BE was wanted unaltered, the chaff density should be changed correspondingly.
\end{abstract}

\section{Introduction}

Chaff Corridor(CC) blanketing effects has been depressed or impaired with developments of radar technology, it holds good as one of important blanket jamming methods so far when air attacks assault designated radars, as CC is easy to extend and to use, low cost, and have universal blanket jamming effects to lots and kinds of radars. Most technologies which confront CC are owing to Doppler effects of chaffs and real targets for radar, so methods and means were tried and considered to re-secure CC's Blanket Efficiency (BE), Such as CC jamming principles in [1][2], chaff mechanical moving characters in atmospheres in [3], basic jamming theory and laying modes in [4], tactical application and its impression in distinct occasions, measure for measure in [5]-[8], intrinsic attenuation characteristics for kinds of radar waves in [9]-[15]. But all of above References were keep same implicit foothold, a precondition that radar's beam was un-considered. But in fact radar's beam is a real material and has its own shape, which means number of chaffs that scatter electromagnetic signals was limited and restricted, so the CC's blanketing theory should be reanalyzed and modified.

\section{Traditional Chaff Corridor}

A CC using scenario is given in Fig.1, to cover the plane $\boldsymbol{T}$, chaffs with given frequencycharacters were scattered abundantly to form CC in atmospheres, which can blanket $\boldsymbol{T}$ from Radar $\boldsymbol{R}$. And the path of electromagnetic waves of radar was not vertical, but acclivitous relative to the jamming CC. The echo received by radar can be sorted into two kinds in Fig.1, one echo was direct rebound from $\boldsymbol{T}$, which was expressed as $\boldsymbol{P}_{\boldsymbol{R} \boldsymbol{R} \boldsymbol{R}}$, the nother was direct rebound from chaff, which was expressed as $\boldsymbol{P}_{\boldsymbol{R} C \boldsymbol{R}}$. Blanket Coefficient(BC) is defined to scale CC's BE as $\boldsymbol{k}_{\boldsymbol{C T}}=\boldsymbol{P}_{\boldsymbol{R} \boldsymbol{C R}} / \boldsymbol{P}_{\boldsymbol{R T R}}$, According to [1] and [2], $\boldsymbol{P}_{\boldsymbol{R} T \boldsymbol{R}}, \boldsymbol{P}_{\boldsymbol{R} \boldsymbol{C} \boldsymbol{R}}$ and $\boldsymbol{k}_{\boldsymbol{C} \boldsymbol{T}}$ can be expressed as Eq. 1, Eq. 2 and Eq. 3. Comparative BC (CBC) was adopted in [16] where pointed the effects of radio skew transmission should be considered in. there was a common Equation as Eq. 4, at beginning was Eq. 5, CBC is defined as Eq. 6. According to Fig.1, and a define was given as Eq. 7 and Eq. 8, at least CBC was expressed as Eq. 9. 


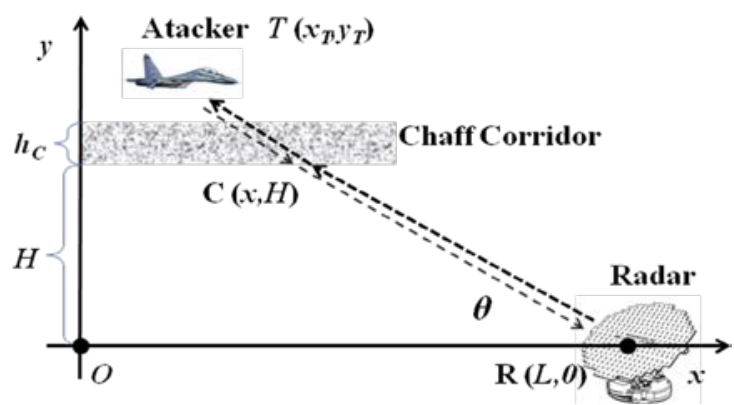

Figure 1 Common CC Jamming Sketch

$$
\begin{aligned}
& P_{R T R}=\exp \left[-0.34 n \lambda^{2} h_{c}\right] P_{R} G_{R}^{2} \sigma_{T} \lambda^{2} /\left[(4 \pi)^{3} R_{R T}^{4}\right] \\
& P_{R C R}=P_{R} G_{R}^{2} \sigma_{C} \lambda^{2} /\left[(4 \pi)^{3} R_{R C}^{4}\right] \\
& k_{C T}=\exp \left(0.34 n \lambda^{2} h_{c}\right) \sigma_{C} / \sigma_{T}, \quad R_{R T} \approx R_{R C} \\
& k_{C T}=\frac{0.17\left(2 H+h_{c}\right) h_{c} \theta_{0.5 H} \theta_{0.5 V} R_{R C}^{2} n \lambda^{2}}{2 \sigma_{T} H^{2}} e^{0.34 \frac{n \lambda^{2} h_{c}}{\sin \theta}} \\
& k_{C T 0}=\frac{0.17\left(2 H+h_{c}\right) h_{c} \theta_{0.5 H} \theta_{0.5 V} R_{R C 0}^{2} n_{0} \lambda^{2}}{2 \sigma_{T 0} H^{2}} e^{0.34 \frac{n_{0} \lambda^{2} h_{c}}{\sin \theta_{0}}} \\
& k=k_{C T} / k_{C T 0}, \sigma_{T} \approx \sigma_{T 0} \\
& k_{1}=R_{R C}^{2} / R_{R C 0}^{2} \\
& k_{2}=\exp \left[0.34\left(n R_{R C}-n_{0} R_{R C 0}\right) \lambda^{2} h_{c} / H\right] \\
& k=k_{1} k_{2}
\end{aligned}
$$

Where from Eq. 1 to Eq. $7 \boldsymbol{P}_{\boldsymbol{R}}$ is radar radiant power, $\boldsymbol{G}_{\boldsymbol{R}}$ is the gain of the radar's antennas, $\boldsymbol{\sigma}_{\boldsymbol{T}}$ is target's equivalent Radar-Cross-Section (RCS), $\lambda$ is radar RF wavelength. $\boldsymbol{R}_{\boldsymbol{R} T}$ is range between radar and target, $\boldsymbol{R}_{\boldsymbol{R} C}$ is range between radar and chaffs, $\sigma_{C}$ is chaffs' $\boldsymbol{R} \boldsymbol{C S}$ in one radar resolution cell, $\boldsymbol{n}$ is CC's chaff density, $\boldsymbol{h}_{\boldsymbol{C}}$ is CC's thickness, and $\boldsymbol{H}$, highness, $\boldsymbol{\theta}$ is the main lobe beam's vertical angle, beam horizontal half width was $\boldsymbol{\theta}_{\mathbf{0 . 5 H}}$, beam vertical half width was $\boldsymbol{\theta}_{\mathbf{0 . 5 V}}, R_{\boldsymbol{R} \boldsymbol{C} \boldsymbol{0}}$ is range between radar and CC's beginning. Also $\boldsymbol{L}$ is defined as radar's location,which will be used later.

\section{Beam Cutting Effect}

\section{A. Beam Cutting of Main Lobe}

Radar main beam was often considered in use, which was unconsidered before from [1] to [15]. In fact in Fig. 2, it had great effect to CC and should be considered as it meaned that there were only part of CC has jamming effect and effective, so chaffs' number was limited in CC.n in Eq. 6 means chaff number which scattered electromagnetic power and in the cross part of radars beam. There was $\boldsymbol{n} / \boldsymbol{n}_{\mathbf{0}}$ in Eq. 7 which was missed. According to Eq. 7 and Fig. 2, an obviously corrective conclusion about $\boldsymbol{n}$ or $\boldsymbol{n}_{\mathbf{0}}$ should be inferred as Eq. 10 .

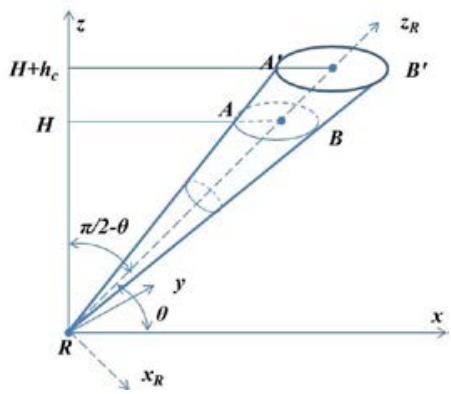

Figure 2 Main Lobe Beam Cutting Sketch

$$
n=n_{c} V_{c}
$$


Where $\boldsymbol{n}_{\boldsymbol{c}}$ means real density of CC, and $\boldsymbol{V}_{\boldsymbol{c}}$ stands for volume of beam cutting shape. Eq. 10 means $\boldsymbol{n} / \boldsymbol{n}_{\mathbf{0}}$ was related with two factors, one was the radar beam cutting space in CC, the other was chaff thickness. So Eq. 9 should be modified as Eq. 11.

$$
\begin{aligned}
& k=k_{0} k_{1} k_{2} k_{3} \\
& k_{0}=n_{c} / n_{c 0} \\
& k_{3}=V_{c} / V_{c 0}
\end{aligned}
$$

\section{B. Volume of Beam Cutting}

According to the stereoscopic relationship in Fig.2, After a series of Mathematical derivation combined with Fig.1, the volume of beam cutting should be expressed as Eq. 14. Also there was a relation as Eq. 15 in Fig.1, there was a relationship, $\boldsymbol{k}_{\mathbf{3}}$ could be introduced out as Eq. 16. Also $\boldsymbol{k}_{\mathbf{2}}$ could be modified as Eq. 17. Equation 17 means that if $\boldsymbol{k}_{\mathbf{0}}$ was changed, $\boldsymbol{k}_{\mathbf{2}}$ would be changed too, also according to the stereoscopic relationship, Eq. 11 should be modified as Eq. 18.

$$
\begin{aligned}
& V_{C}=\frac{\left(H^{2} h_{C}+H h_{C}^{2}+h_{C}^{3} / 3\right) \pi t g \theta_{0.5 V} \operatorname{tg} \theta_{0.5 H}}{\left(\sin ^{2} \theta-\cos ^{2} \theta \cdot \operatorname{tg}^{2} \theta_{0.5 V}\right)^{1.5}} \\
& \left\{\begin{array}{l}
\sin ^{2} \theta=H^{2} /\left[H^{2}+(L-x)^{2}\right] \\
\cos ^{2} \theta=(L-x)^{2} /\left[H^{2}+(L-x)^{2}\right]
\end{array}\right. \\
& k_{3}=\frac{V_{C}}{V_{C 0}}=\frac{\left[H^{2}+(L-x)^{2}\right]^{1.5}\left[H^{2}-L^{2} \operatorname{tg}^{2} \theta_{0.5 V}\right]^{1.5}}{\left[H^{2}+L^{2}\right]^{1.5}\left[H^{2}-(L-x)^{2} \operatorname{tg}^{2} \theta_{0.5 V}\right]^{1.5}} \\
& k_{2}=\exp \left[0.34\left(k_{0} R_{R C}-R_{R C 0}\right) n_{0} \lambda^{2} h_{c} / H\right] \\
& k=k_{0} k_{1} k_{2}\left(k_{0}\right) k_{3}
\end{aligned}
$$

\section{Reference of $\mathbf{C}$}

According to [2], if radio waves power is attenuated to a degree of $\Delta p(d B)$ for CC, there was a ralation as Eq. 19, the original chaff density of CC could be introduced as Eq. 20. As mentioned above, radio waves power is sanded and intercepted by radar in a skew path relative to CC, so Eq. 20 should be modified as Eq. 21.

$$
\begin{aligned}
& \Delta p=2 \beta h_{C}, \beta=0.738 n_{0} \lambda^{2} \\
& n_{0}=\Delta p /\left[1.476 h_{C} \lambda^{2}\right] \\
& n_{0}=\Delta p \sin \theta /\left[1.476 h_{C} \lambda^{2}\right]
\end{aligned}
$$

Combined with Eq. 5, the reference BC. of the corridor beginning could be drowed out.

\section{Comparative Simulation}

Assuming a CC was scattered to jam to the radar, where $\boldsymbol{L}=100 \mathrm{~km}, \boldsymbol{H}=6 \mathrm{~km}, \boldsymbol{h}_{\boldsymbol{c}}=0.5 \mathrm{~km}$, $\lambda=5 \mathrm{~cm}$, the RCS of taget was $15 \mathrm{~m}^{2}$, for a given attenuation degree of $\boldsymbol{\Delta} \boldsymbol{p}=\mathbf{6 0 d B}$, according to Eq. 24 and Eq. 3, the initial chaff density and the wanted BC of CC could be got as Eq. 22.

$$
n_{0}=33, k_{C T}=0.0009
$$

In such case, in fact the real BC was Eq. 23 according to Eq. 5, and the initial chaff density according to Eq. 21, the CC's BC according to Eq. 5.

$$
k_{\text {CT }}=25.76, n_{0}=3, k_{\text {Ст } 0}=1.893
$$

Modified initial chaff density was less than the traditional's, which mean that mass chaffs could be saved.

\section{A. Simulation of Even CC}

By all appearances, if the CC has a even chaff density from cover to cover, the closer from CC's position to radar, the smaller CBC $\boldsymbol{k}$ will be. For the given scenario of Fig.1, $\boldsymbol{k}$ were showed in

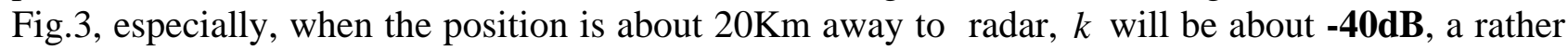
small value. 


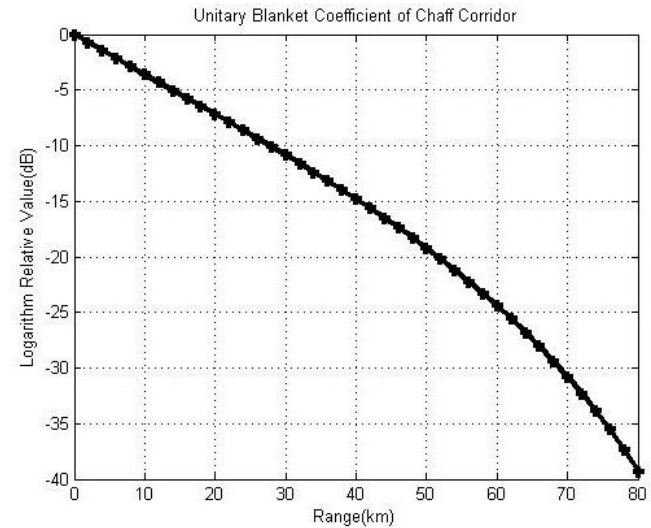

Figure 3 CBC Logarithm Curve
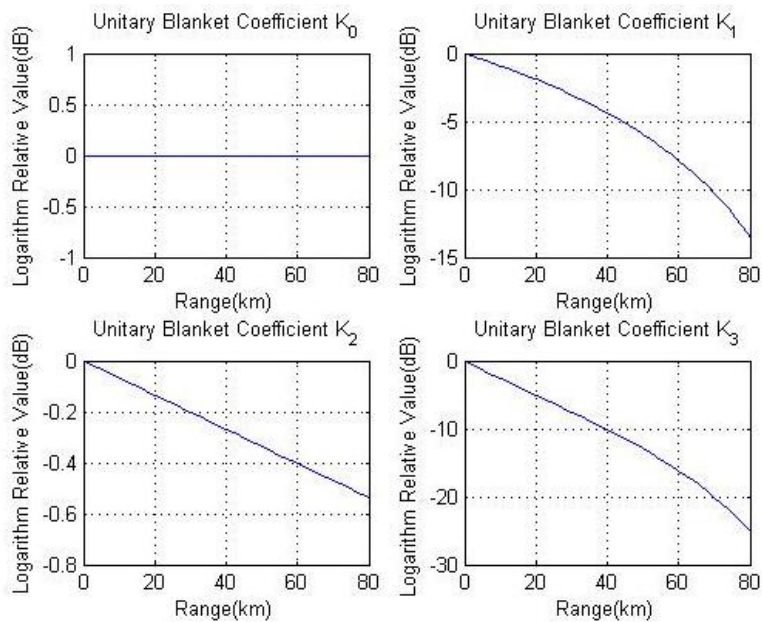

Figure 4 parameters of $\mathrm{k}$ in logarithm

Sub-parameters were showed in Fig.4 according to Eq. 12, Eq. 7, Eq. 17 and Eq. 16. Though $\boldsymbol{k}_{0}$ was unchanged, others were attenuated, especially $\boldsymbol{k}_{\mathbf{1}}$ and $\boldsymbol{k}_{\mathbf{3}}$ were decayed greatly.In such status, if such an even CC is laid, the needed chaff number should be figured out firstly. If it was figured out according to CC's end position nearest radar, mass of chaffs will be waste; otherwise if it was figured out according to CC's begin position far from radar, CC's BC for radar will not be assured.

\section{B. Simulation of Un-even CC}

If CC was expected to jam effective from over to over, $k$ should be nearly invariable, which means that CC's thickness or chaff density should be altered. As aeroplanes which scatter chaffs to form jamming CC usually fly at a constant velocity, chaff thickness is hard to customized relatively, but chaff density is reversely easy. The latter way was selected to change $k$, in another word, the sub-parameter $\boldsymbol{k}_{\mathbf{0}}$ should be changed. But according to Eq. 17, if $\boldsymbol{k}_{\boldsymbol{0}}$ was changed, $\boldsymbol{k}_{\mathbf{2}}$ was changed spontaneously too. So a testing by testing way was adopted to make the $k$ keep common and even by Eq. 21. The results of sub-parameters was showed in Fig. 5.
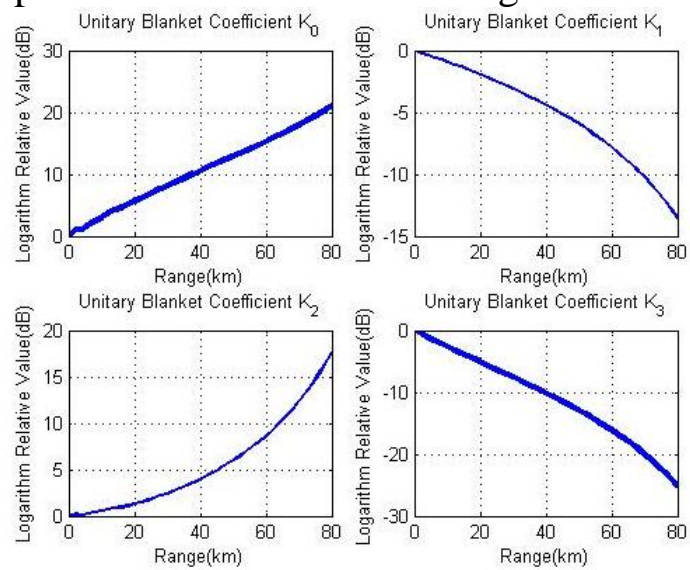

Figure 5 improved Parameters of $\mathrm{k}$ in logarithm 
$k$ is showed in Fig.6, which keeping constant in the rough. In Fig.5, the $\boldsymbol{k}_{\boldsymbol{0}}$ was almost an oblique line in logarithm, which signified that the chaff density should be promoted in an exponent level.

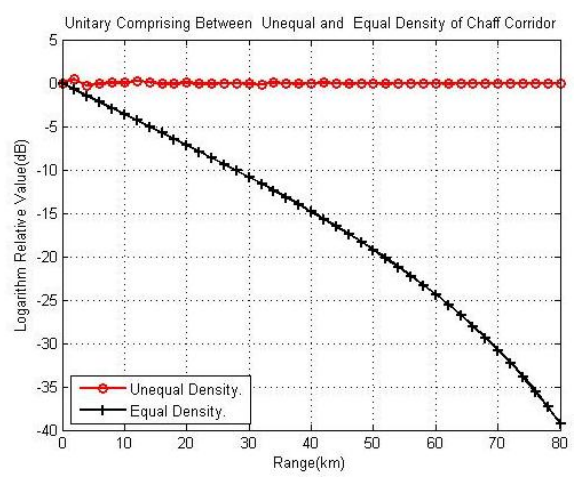

Figure 6 Upgrade CBC Curve

\section{Conclusion}

It must be pointed out that there are many factors that are correlative with CC's BC, and should be considered synthetically in practice. But in any case, the chaff density is also one of importants for CC's BC, which was not concerned before. CC has distributed BC. Similar analysis can be carry out in the before-mentioned way in situation such as laying side CC to radar, or jamming two or more radars, and results will be likeness. This is important to upgrade CC's BC, and will promote CC’s performance in practice.

\section{References}

[1] Lin Xiang-ping, "Radar Countermeasure Theory”. Xi'an: Publishing House of Northwest Telecommunication Engineer College, pp.121-122, 1985.

[2] Wang Xing,“Airborne Countermeasure Countermeasure Theory”. Beijing: National Defense Industry Press, pp.371-378,2008.

[3] Cai Wan-yong,Li Xia and Wan Shan-hu, "Model of chaff motion trajectory and curtain wall diffusion in air environ- ment” [J],Systems Engineering and Electronics, pp. 565-569, Mar.2009.

[4] Sun Wei-tong and Jiang Feng, "Research on Jamming Corridor”, Electro-Optic Technology Application, pp.28 31, Dec.2005.

[5] Gan Yuan-Liu, “Actuality and Development of Jamming Corridor”, Electro-Optic \&Passive Counter-Measure, pp.1 3, Apr.1999.

[6] Cai Gan, Zhang Ke and Li Yan-jun, “Tactical Application Research of Chaff Corridor”, Fire Control \& Command Control, pp.46 47, July, 2008.

[7] Yang Da-hai, Ma De-bao and Feng Zhi-hong. "Chaff Corridor Interference Analysis of the Space-borne SAR”, ELECTRO- NIC WARFARE, pp.22-25, Jan.2010.

[8] Feng De-jun ,Wang Xue-song and Liu Jia-qi, “Jamming Effect Analysis of Chaff Cloud on Ground-based Radar and Its Application”, Modern Radar, pp.22-26, Jun.2010.

[9] Su Fu, Wang Xiao-nian and Pi Jun-ming, "Simulation and Analysis of Jamming Effectiveness of Jamming Corridor on Ground Early Warning Radar”, Fire-Control Radar Technology, pp.57-60, Sept.2007.

[10]Chen Jing, “There is No Relationship between the function of Jamming Corridor and Radar System”, ELECTRO-OPTIC TECHNOLOGY APPLICATION, pp.38 42, Aug.2005. 
[11]Hu Song, Lu Jing-shuang and Xu Tong, "Effect Evaluation of Chaff Suppress Jamming on Power Attenuation of Radar Signal”, Journal of Jilin University (Information Science Edition), pp.259-263, May 2008.

[12] Hu Song, Lu Jing-shuang and Li Yan-zhi, "Evaluation of Operation Effectiveness of Chaff Corridor Covering Airplane Formation Penetration”, SHIPBOARD ELECTRONIC COUNTER- MEASURE, pp.20 23, Oct.2008.

[13] Lu Jing-shuang ,Hu Song and Zhou Rui, "Research into The Jamming Eff iciency of Corridor Chaff to MTI Radar”, SHIP- BOARD ELECTRONIC COUNTERMEASURE, pp. 31-33, Aug. 2009.

[14]Zhang Yun-fei, Zhang Kao and He Qing-zhi, "Influence Analysis and Calculation of Chaff Corridor on The Stelth Performance of Aircraft", ACTA AERONAUTICA ET ASTRONAUTICA SINICA, pp.202-205, May 2000.

[15]Wang Hong-xun, Ye Guang-qiang, Wang Hong-wei and Qiao Qing-Gang. "Unequal Density Chaff Corridor and its Blanket Effect Upgrade”, Telecommunication Engineering, pp.35-39, April.2011. 